\title{
Industrial Qualification Process for Optical Fibers Distributed Strain and Temperature Sensing in Nuclear Waste Repositories
}

\author{
S. Delepine-Lesoille, ${ }^{1}$ X. Phéron, ${ }^{1}{ }^{J}$. Bertrand, ${ }^{1}$ G. Pilorget, ${ }^{1}$ G. Hermand, ${ }^{1}$ R. Farhoud, ${ }^{1}$ \\ Y. Ouerdane, ${ }^{2}$ A. Boukenter, ${ }^{2}$ S. Girard, ${ }^{2}$ L. Lablonde, ${ }^{3}$ D. Sporea, ${ }^{4}$ and V. Lanticq ${ }^{5}$ \\ ${ }^{1}$ National Radioactive Waste Management Agency (Andra), 1-7 rue Jean Monnet, Parc de la Croix blanche, \\ 92298 Chatenay-Malabry, France \\ ${ }^{2}$ LabHC, UMR CNRS 5516, Université de Saint-Etienne, 42023 Saint-Etienne, France \\ ${ }^{3}$ iXFIber, 22300 Lannion, France \\ ${ }^{4}$ National Institute for Laser, Plasma and Radiation Physics, Magurele, Romania \\ ${ }^{5}$ Cementys, 27 Villa Daviel, 75013 Paris, France
}

Correspondence should be addressed to S. Delepine-Lesoille, sylvie.lesoille@andra.fr

Received 20 July 2012; Accepted 8 October 2012

Academic Editor: Qiang Wu

Copyright (C) 2012 S. Delepine-Lesoille et al. This is an open access article distributed under the Creative Commons Attribution License, which permits unrestricted use, distribution, and reproduction in any medium, provided the original work is properly cited.

\begin{abstract}
Temperature and strain monitoring will be implemented in the envisioned French geological repository for high- and intermediate-level long-lived nuclear wastes. Raman and Brillouin scatterings in optical fibers are efficient industrial methods to provide distributed temperature and strain measurements. Gamma radiation and hydrogen release from nuclear wastes can however affect the measurements. An industrial qualification process is successfully proposed and implemented. Induced measurement uncertainties and their physical origins are quantified. The optical fiber composition influence is assessed. Based on radiation-hard fibers and carbon-primary coatings, we showed that the proposed system can provide accurate temperature and strain measurements up to $0.5 \mathrm{MGy}$ and $100 \%$ hydrogen concentration in the atmosphere, over $200 \mathrm{~m}$ distance range. The selected system was successfully implemented in the Andra underground laboratory, in one-to-one scale mockup of future cells, into concrete liners. We demonstrated the efficiency of simultaneous Raman and Brillouin scattering measurements to provide both strain and temperature distributed measurements. We showed that $1.3 \mu \mathrm{m}$ working wavelength is in favor of hazardous environment monitoring.
\end{abstract}

\section{Introduction}

Distributed optical fiber sensors (OFSs) [1-3] are a key technology for the monitoring of the planned French deep geological repository for long-lived high-level and intermediatelevel wastes, called Cigéo. Temperature and strain distributed sensing based on Raman, Rayleigh, and Brillouin scatterings offer exceptional advantages over traditional electronic sensors, especially as they provide distributed data over the entire structure and thus overcome limitations of traditional sensors, whose information is restricted to local effects.

This paper focuses on temperature and strain distributed sensing based on Raman and Brillouin scatterings in optical fibers for structural health monitoring, more precisely for nuclear industry. Although commercial off-the-shelf sensors and interrogation units are numerous, the global measuring chain may provide disappointing monitoring results to the end-users, unless a number of considerations specific to nuclear environments are taken into account. These are further developed within this paper, with an emphasis on environmental conditions influence, especially (i) temperature, (ii) gamma rays, and (iii) hydrogen influences.

Andra's (French National Radioactive Waste Management Agency) potential applications include surface and deep geological radioactive waste disposal structure monitoring, for instance within the future geological repository that would contain highly instrumented disposal cells. Intermediate-level long-lived waste cells are presently 
designed as $400 \mathrm{~m}$ long tunnels, with a $1 \mathrm{~m}$ thick concrete liner, placed $500 \mathrm{~m}$ deep in a clay rock called CallovianOxfordian formation.

Monitoring aims at preserving retrievability of nuclear wastes, assessing long-term safety, enabling optimization of structures all along the exploitation which is expected to last a century. A major specification of the geological repository monitoring system is durability, required to last up to a century, despite hazardous conditions: gamma rays and hydrogen release. Because of high gamma radiation doses, disposal cells are not accessible as soon as exploitation starts, and the first nuclear waste package is placed inside the disposal cell. This implies the monitoring system to be robust for decades without any maintenance. OFS are a robust technology known to handle radiations quite well. Durability is also ensured thanks to their ability to perform remote sensing, which enables maintenance of optoelectronic instruments. Finally, OFSs are very attractive for their small size and varied external coatings, which limits the preferential flow paths and reduces invasiveness, a highly important aspect to avoid affecting the long-term safety of the geological repository for nuclear wastes.

For these attractive advantages, Andra drives many research studies on distributed temperature and strain sensing with optical fiber sensors. To ensure measurement quality and lifetime of the global monitoring system, Andra has implemented a qualification procedure, which will be presented in the first part of the paper. Previous reported results gathered within this qualification process will be recalled. The second part focuses on recent results: field test where temperature compensation of strain measurements, based on combined Brillouin and Raman scatterings, was successfully implemented. The third part presents research results on hazardous condition influences on both Brillouin and Raman scatterings as a function of optical fiber types. We conclude with a recommendation on the optimized sensing system for our application.

\section{Qualification Procedure and Previous Results}

2.1. Qualification Procedure. The outstanding properties of optical fiber sensors drove major interest in structural health monitoring applications. Nevertheless, it is important to notice that optical fiber and optical installation practices used in telecommunications and other industries are significantly different from nuclear constraints and applications, which may be far less tolerant.

Moreover, optical fiber sensing systems presently suffer from a lack of standardization of claimed performances. Dedicated qualification processes are not defined yet. Andra has implemented a multistage qualification procedure for each selected measurement chain. For OFS, it was chosen to study both sensing cables and optoelectronic instruments separately before pairing such elements and focusing on data processing.

The described overall process is inspired from [4]. Global test sequence includes four stages.
Stage one consists in acquiring in-depth knowledge of the sensing technology, engineering solutions, and practical implementation constraints. It aims at selecting the technologies best suited to the specific requirements of monitoring the geological repositories for long-lived nuclear wastes. When off-the-shelf sensing chain performances do not fulfil requirements, Andra initiates research programs. It has been the case for distributed optical fiber sensing system whose results are presented in this paper.

Stage two consists in carrying out laboratory tests, under fully supervised and/or controlled environmental conditions, to qualify the sensitive component and assess the complete measurement chain performances. Sensors are tested alone, then embedded in the host material of interest.

Stage three consists in outdoor tests, to evaluate field implementation influence. At this stage, the sensing chain is preserved from hazardous conditions, extreme temperature, or gamma rays. Unexpected influence of various parameters might thus be revealed.

The fourth stage involves hardening in view of the application environmental conditions. In the envisioned French geological repository, temperature would range from $20^{\circ} \mathrm{C}$ to $90^{\circ} \mathrm{C}$. Gamma radiation rates reach $1 \mathrm{~Gy} / \mathrm{h}$, total dose $10^{7} \mathrm{~Gy}$. Hydrogen release is also expected; its maximum levels could approach $100 \%$ hydrogen content in the atmosphere.

2.2. Previous Results. This qualification methodology has been implemented for distributed temperature and strain sensing.

Andra selected Raman scattering to perform distributed temperature measurements, as it is the most advanced technology with superior temperature sensitivity, better than $0.1^{\circ} \mathrm{C}$ [5]. Andra selected Brillouin scattering for distributed strain sensing, since interferometric measurements are in favor of durability. The first three stages of the qualification procedure, laboratory tests, and preliminary outdoor tests were reported in [6]. Two remaining questions were to be addressed to end the qualification procedure.

First, how to compensate for Brillouin scattering temperature sensitivity? The Brillouin frequency shifts are known to be proportional to temperature $(\Delta T)$ and strain $(\varepsilon)$ variations as in (1) [7]:

$$
\Delta \nu_{B}=C_{T} \Delta T+C_{\varepsilon} \varepsilon .
$$

$C_{T}$ and $C_{\varepsilon}$ are characteristics of the optical fiber type. At the operating wavelength $(1550 \mathrm{~nm})$, for standard G652 singlemode fiber, $C_{T}$ and $C_{\varepsilon}$ are in the order of $1 \mathrm{MHz} /{ }^{\circ} \mathrm{C}$ and $0.05 \mathrm{MHz} / \mu \varepsilon$ [8]. Instruments based on Brillouin scattering would perform either temperature or strain measurements; strain is 20 times less influent than temperature. A solution to decorrelate strain from temperature is detailed in Section 3.

Second, what are hydrogen and gamma rays influences on Raman and Brillouin scatterings? Quantitative evaluation of such influence as a function of optical fiber types is presented in Section 4. Based on these various evaluations, a last paragraph predicts lifetime of the optical fiber geological repository monitoring system, anticipating strain and temperature measurements uncertainties and distance range. 


\section{Tunnel Liner Instrumentation: Strain Measurement Temperature Compensation}

Andra has created an Underground Research Laboratory to evaluate the constructability, safety, and reversibility of the potential radioactive waste disposal in the CallovianOxfordian clay stones. A multidisciplinary program is implemented in this $500 \mathrm{~m}$ underground structure. The "retaining and covering observation experiment" is taking place into a dedicated gallery with a concrete liner (Figure 1), similar with intermediate-level long-lived waste disposal cells. Its purpose is to evaluate several monitoring solutions. This experiment integrates onfield constrains (drilling environment, dust, and operational phases with limited intervention time) and a representative experiment in view of future geological repository (one-to-one scale, specific rock in its natural location).

3.1. Instrumented Structure. The gallery is $5 \mathrm{~m}$ in diameter with a $50 \mathrm{~cm}$ thick concrete liner. Many parameters were monitored in both clay stones and covering concrete: temperature, strain, water content, and interstitial fluid pressure.

One section of the concrete liner was instrumented with collocated fiber optic sensing cables whose picture is illustrated in Figure 2.

Andra had previously tested Raman temperature sensing into single-mode fibers in a surface building slab. This test highlighted the great sensitivity of Raman scattering in single-mode fiber to curvature, which can poorly be avoided in civil engineering structures [9]. This is why two different sensing lines were implemented: multimode fibers were used for Raman temperature monitoring and single-mode fibers were installed for Brillouin strain sensing. More precisely, we used three different sensing cables, two for Brillouin measurements, composed of G652 and G657 fiber types to evaluate curvature sensitivity, and one for Raman sensing. The three cables were placed redundantly so that, finally, six sensing arches were embedded inside the concrete liner.

In order to ensure accurate positioning and maintaining during concrete pouring, sensing cables were attached to a thin piece of wire mesh, spitted on the retaining concrete.

Measurements were remotely performed every $15 \mathrm{~min}$ utes by commercially available instruments, a Brillouin OTDA (optical time-domain analyser) and a Raman distributed temperature sensing (DTS). Both devices were set at $0.5 \mathrm{~m}$ spatial resolution.

Instruments were located in another gallery of the underground laboratory. The full sensing line is approximately $500 \mathrm{~m}$, with only $20 \mathrm{~m}$ embedded inside the concrete liner.

Electronics sensors, such as vibrating wire extensometers and platinium probes, were colocated with optical fiber sensors.

3.2. Results of Early Age Monitoring. The gallery liner was constructed in 2 steps. First, the bottom part (inverted arch) concrete was poured. One month after, the second arch completed the liner.

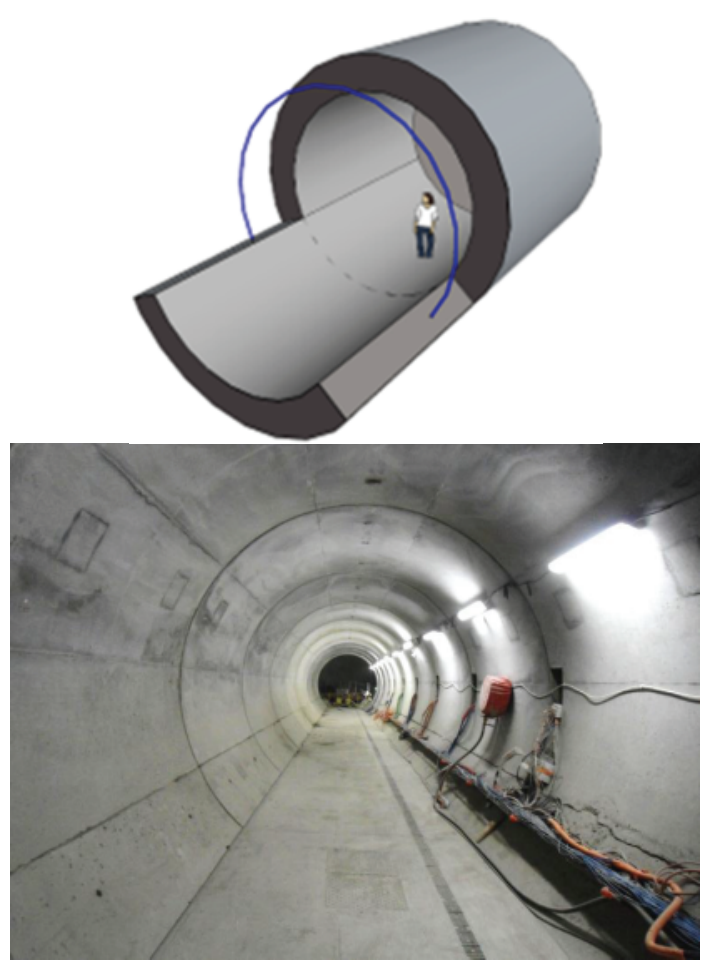

FIGURE 1: Scheme of gallery instrumentation by distributed sensing and picture of the gallery 6 months after liner casting.

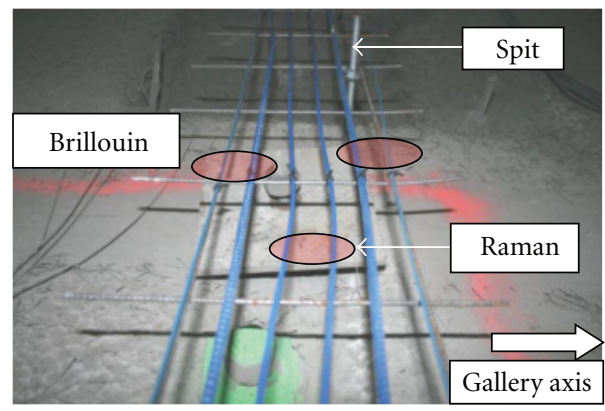

Figure 2: Picture of optical fiber cables installed on a gallery circumference, before concrete casting.

One from the six sensing lines got damaged during construction. We did not observe any advantage of G652 compared with G657 on the strain measurement quality.

During concrete hardening, an exothermal chemical reaction takes place. Consequently, temperature increases and Brillouin frequency shifts. Thermal expansion of concrete induces strain on the optical fiber cable, which also increases Brillouin frequency. Temperature measurement acquired by Raman sensing lines (left $y$ axis) and raw Brillouin frequency (right $y$ axis) are illustrated in Figure 3.

To provide useful information, concrete thermal expansion must be differentiated from the parameters of interest: strain induced by stress, creep, and shrinkage, noted $\varepsilon_{\text {comp }}$ (comp. stands for "compensated"). If $\alpha_{\text {concrete }}$ is the concrete 


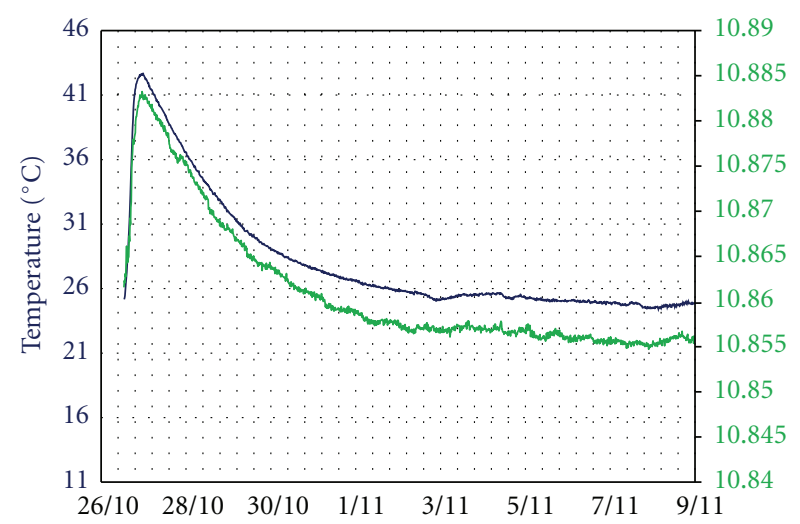

FIgURE 3: Raman temperature measurement and Brillouin frequency shift during concrete hardening.

thermal expansion coefficient, temperature-compensated strain can be obtained following equation (2):

$$
\varepsilon_{\text {comp }}=\frac{\Delta \nu_{B}}{C_{\varepsilon}}-\frac{\left(C_{T}+\alpha_{\text {concrete }} C_{\varepsilon}\right) \cdot \Delta T}{C_{\varepsilon}} .
$$

Embedded and instrumented concrete samples [10] enabled in situ measurement of $\alpha_{\text {concrete }}=10 \mu \varepsilon /{ }^{\circ} \mathrm{C}$. Assuming $C_{T}=1 \mathrm{MHz} /{ }^{\circ} \mathrm{C}$ and $C_{\varepsilon}=0.05 \mathrm{MHz} / \mu \varepsilon$, we obtained the compensated strain measurements plotted in Figure 4.

The $150 \mu \mathrm{m} / \mathrm{m}$ compressive strain measured is the consequence of the early-age shrinkage of concrete. This value is fully consistent with the one measured by the vibrating wire extensometers placed nearby the optical fibers.

Uncertainties were evaluated analyzing 24 repeated measurements acquired during $6 \mathrm{~h}$ (inset Figure 4). Such analysis has been repeated. We obtain uncertainty in the order of $30 \mu \mathrm{m} / \mathrm{m}$, which corresponds to the Brillouin instrument performance. Temperature uncertainty was better than $0.2^{\circ} \mathrm{C}$ in the Raman sensing line (Figure 3). The Raman-Brillouin compensation method does not increase the initial strain and temperature measuring system uncertainties.

Our results were obtained with $500 \mathrm{~m}$ distance range and a $0.5 \mathrm{~m}$ spatial resolution. Similar experiments of Raman temperature compensated Brillouin strain measurements reported $3.6^{\circ} \mathrm{C}$ and $80 \mu \mathrm{m} / \mathrm{m}$ repeatability with $5 \mathrm{~m}$ resolution [11].

This method also enables long-term monitoring. Concrete liner strain evolution has been acquired all along construction steps; measurements will go on in the next years to acquire as much information as possible before the repository construction.

An advantage of distributed measurements provided by optical fibers is illustrated in Figure 5. In this gallery, covering concrete is not reinforced (no steel bar). Tensile strain zones were identified and precisely located by distributed measurements at locations where there was no electronic sensor.

As conclusion, a sensing scheme combining Raman and Brillouin instruments with multiple sensing cables is a promising solution for performing simultaneous temperature and strain monitoring.

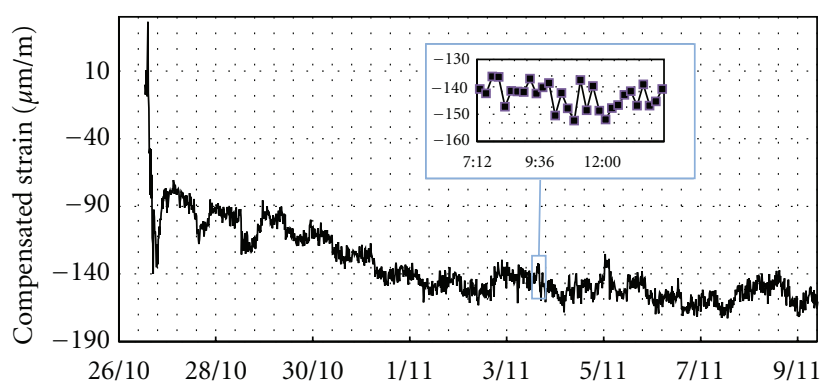

FIGURE 4: Processed data: temperature compensated strain acquired during concrete pouring ( $x$ axis is dates, from October to November 2011).

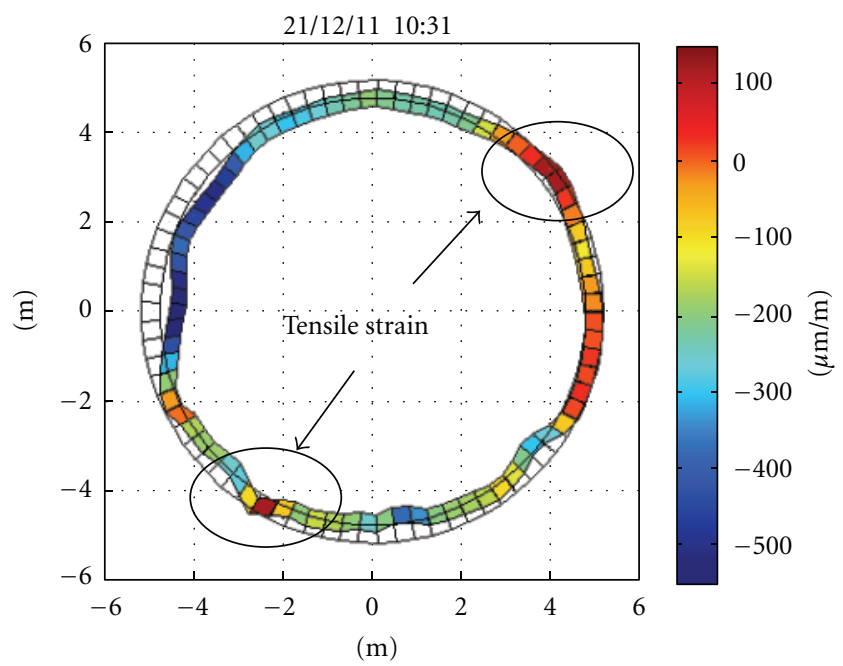

Figure 5: Compensated strain reported on section after arch construction.

This real-scale experiment demonstrates that a RamanBrillouin temperature and strain sensing is very well suited for underground tunnel monitoring. It also appeared that chosen optical fiber cables were robust enough to put up with construction conditions.

\section{Compatibility with Future Hazardous Environments}

Nuclear waste repository is a challenging environment due to the presence of gamma radiations which is known to degrade the optical properties of fibers through three different phenomena [12]: radiation-induced attenuation (RIA) decreases fiber transmission efficiency, radiationinduced emission (RIE) decreases signal-to-noise ratio and compaction changes refractive index (especially for very high particle fluences or doses).

The amplitudes and kinetics of these changes depend on many parameters, among which are dose rates, total doses, optical fiber type, and operating wavelength. 


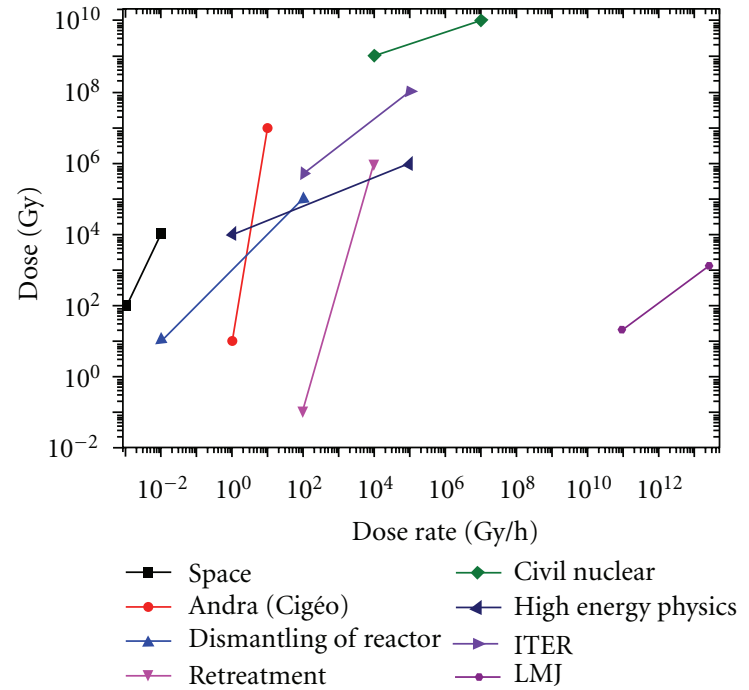

Figure 6: Overview of typical applications as a function of total dose and dose rate [13].

As illustrated in Figure 6, in Andra geological repository, doses rates are moderate; however, after 100 years of monitoring in the vicinity of high-level wastes structure cells, total doses will reach $10^{7} \mathrm{~Gy}$.

4.1. Gamma Influence on Brillouin Scattering. It has been demonstrated on standard G652 fibers that there is a limited influence of gamma rays on the Brillouin frequency at least up to doses of $100 \mathrm{kGy}$ [14]. To evaluate possible influence at total doses corresponding to our application, we performed in situ [15] and postmortem gamma-ray irradiation tests in different classes of fibers.

Gamma-ray irradiations were performed at room temperature using the Brigitte facility, cobalt-60 source, at SCKCEN (Belgium) [16], with a dose rate of $\sim 28 \mathrm{kGy} \cdot \mathrm{h}^{-1}$.

We selected our samples among the most commonly used fibers, like the SMF28 from Corning, since almost all commercially available Brillouin instruments are designed to operate with this type of fibers. Thus, the first selected fiber was the step-index fiber SMF28 from Corning. In view of the geological repository monitoring requiring high gamma dose tolerance, we selected a fluorine-doped cladding, pure silica-core fiber. The fiber has a $5 \mu \mathrm{m}$ core radius and a $40 \mu \mathrm{m}$ cladding radius with a fluorine concentration of $1.25 \% \mathrm{~mol}$. Finally, to evaluate the influence of high concentrations of dopants, we tested a highly $\mathrm{GeO}_{2}$-doped core fiber at $28 \% \mathrm{~mol}$ concentration with a core radius of $1.4 \mu \mathrm{m}$ and a pure silica cladding with radius of $62.5 \mu \mathrm{m}$ (HGe in the following).

As expected and detailed in Table 1, measured RIA levels are important at the high doses (up to $10^{7} \mathrm{~Gy}$ ), with a factor up to 4 between the RIA measured for the two extreme cases. The most tolerant fiber, the F-doped fiber, quickly saturates, at a moderate dose level of $5 \mathrm{MGy}$, at RIA level around $50 \mathrm{~dB} / \mathrm{km}$. Impact on distance range is discussed later.

For the dose range corresponding to geological repository application, measured central Brillouin frequencies also shifted (BFS) with dose, whatever the considered optical fiber types. Main parameters of the Brillouin spectrum are listed in Table 1 as well as their changes with dose. Unlike SMF28 and HGe fiber, a clear saturation in the Brillouin frequency shift $\left(\Delta \nu_{B}\right)$ occurs at $\sim 2 \mathrm{MGy}$ for the F-doped fiber. This saturation effect is of major interest for the target application where expected doses largely exceed this saturation level dose. Moreover, for this fiber, Brillouin frequency shift remains small, in the order of $2 \mathrm{MHz}$, which corresponds to $2^{\circ} \mathrm{C}$ measurement error or $40 \mu \mathrm{m} / \mathrm{m}$. On the opposite, $18^{\circ} \mathrm{C}$ temperature measurement error would occur if $\mathrm{HGe}$ fiber was selected.

Strain and temperature sensing based on Brillouin scattering involves both the central Brillouin frequency measurement and hypothesis on calibration coefficients $\left(C_{\varepsilon}, C_{T}\right)$ (see (1)). These calibration coefficients might also be impacted by radiation. To our knowledge, it is the first time radiation influence on these parameters is evaluated.

No significant effect of radiations on $\mathrm{C}_{T}$ could be observed for the SMF28 and HGe fibers (Figure 7). For the Fdoped fiber, a decrease of about $6 \%$ was noted in the $\mathrm{C}_{T}$ coefficient after a deposited dose of $10^{7} \mathrm{~Gy} . C_{\varepsilon}$ strain coefficients do not seem to depend on received gamma dose, whatever the fiber type. As a conclusion, gamma impact on calibration factors would increase measurement uncertainties by few percent only.

As a conclusion, radiation effects on the performances of strain and temperature Brillouin scattering based optical fiber sensors have been deeply investigated for different classes of optical fibers. There is a strong influence of the core composition. Thus, optical fiber type should be carefully chosen to ensure durability of the monitoring system.

The presented tests are relevant since it is well known that higher dose rates increase RIA; thus, results overestimate degradations that will endure the geological repository monitoring system.

Fluorine-doped pure-silica core fibers are able to handle dose rates and total doses during one century of monitoring within the geological repository. Strain and temperature measurement uncertainties would slightly degrade. The most impacting radiation effect is RIA. Since optoelectronic instrument optical budget is in the order of $10 \mathrm{~dB}$, distance range would be reduced down to $200 \mathrm{~m}$ for the F-doped fiber after a century of monitoring. It suits requirements as (i) high-level long-lived waste (HLW) disposal cell will be 40 to $100 \mathrm{~m}$ long and (ii) in $400 \mathrm{~m}$ intermediate-level long-lived waste storage cell, doses are greatly reduced.

On the opposite, with SMF28 fiber, distributed sensing would be compromised after only a decade of monitoring along $100 \mathrm{~m}$ of HLW disposal cell, instead of the required century.

We focused our study on the response at the $1550 \mathrm{~nm}$ wavelength since it is the commercial instrument working wavelength. However, a global optimization of the entire system must also consider this parameter as a possible variable. Initially, optical losses are slightly more important in pristine optical fibers at the $1310 \mathrm{~nm}$ wavelength compared to the ones reported at $1550 \mathrm{~nm}$. Yet, after a $10 \mathrm{MGy}$ dose, the fibers exhibit lower propagation losses at $1310 \mathrm{~nm}$; for instance, $23 \mathrm{~dB} / \mathrm{km}$ versus $56 \mathrm{~dB} / \mathrm{km}$ at $1550 \mathrm{~nm}$ for the fluorine fiber. 
TABLE 1: Summary of radiation-induced attenuations and Brillouin frequency shifts.

\begin{tabular}{|c|c|c|c|c|c|c|c|}
\hline & $\nu_{B 0}(\mathrm{MHz})$ & Dose (MGy) & 1.1 & 3 & 5.5 & 7.82 & 10 \\
\hline \multirow{2}{*}{ SMF 28} & \multirow{2}{*}{10843} & $\operatorname{RIA}(\mathrm{dB} / \mathrm{km})$ & 84.8 & 147 & 186 & 214 & 253 \\
\hline & & $\Delta v_{B}(\mathrm{MHz})$ & 1.0 & 1.5 & 2.0 & 3.0 & 4.0 \\
\hline \multirow{2}{*}{ HGe fiber } & \multirow{2}{*}{9276} & RIA (dB/km) & & 270 & & 324 & 406 \\
\hline & & $\Delta \nu_{B}(\mathrm{MHz})$ & & 9.6 & & 13.3 & 17.8 \\
\hline \multirow{2}{*}{ F-fiber } & \multirow{2}{*}{11050} & $\mathrm{RIA}(\mathrm{dB} / \mathrm{km})$ & 25 & 38 & 51 & 54 & 56 \\
\hline & & $\Delta v_{B}(\mathrm{MHz})$ & 0.8 & 2.0 & 2.1 & 2.2 & 2.3 \\
\hline
\end{tabular}

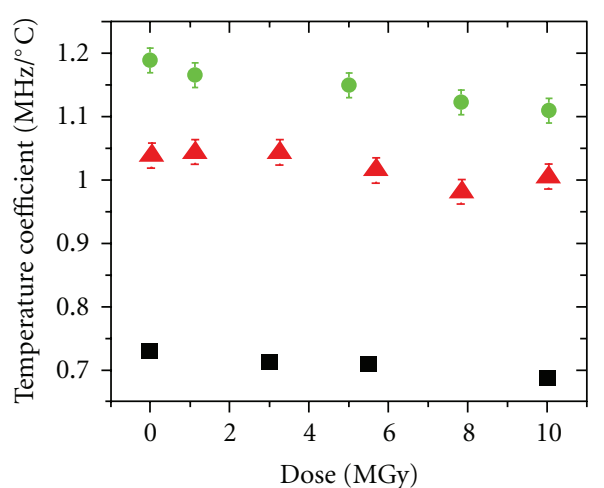

- $\mathrm{HGe}$

- $\mathrm{SiO}_{2} / \mathrm{F}$

- SMF28

(a)

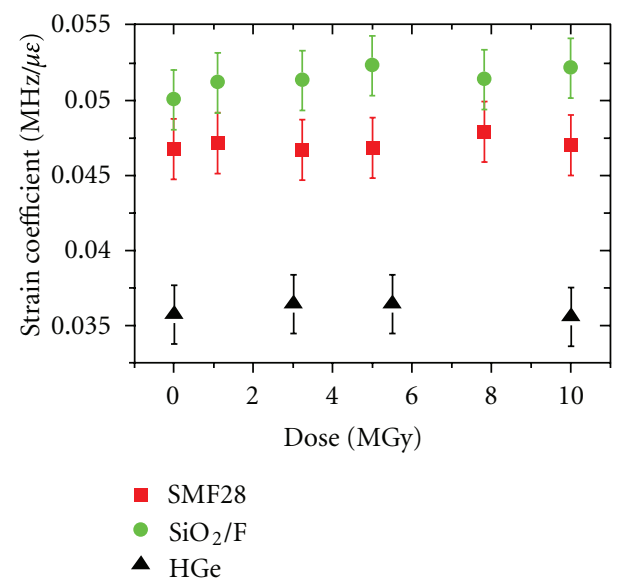

(b)

Figure 7: Temperature $\left(C_{T}\right.$-up) and strain $\left(C_{\varepsilon}\right.$-down $)$ Brillouin calibration coefficients, with received gamma dose for the three optical fiber types.

4.2. Hydrogen Influence on Brillouin Scattering. As introduced previously, for nuclear waste repository instrumentation, hydrogen influence must also be quantified.

Hydrogen originates from (i) nuclear waste release and (ii) anoxic corrosion of metallic materials. Although hydrogen releases are expected small (in the order of $430 \mathrm{mmol} /$ hour release for each intermediate-level nuclear waste), when ventilation stops with cell closure, concentrations would slowly yet regularly increase. Its maximum levels could approach $100 \%$ hydrogen content in the atmosphere in few months.

The chosen samples were the same F-doped and HGe fibers used for radiation studies. A G652 from iXFiber company was chosen as a reference. It is composed of a $\mathrm{GeO}_{2}$-doped core fiber at $3.4 \mathrm{~mol} \%$ concentration with a $4.6 \mu \mathrm{m}$ radius and a pure silica cladding with radius $62.5 \mu \mathrm{m}$.

The optical fibers were placed inside autoclave chambers, where molecular hydrogen pressure was maintained at 150 bars and temperature was regulated at $25^{\circ} \mathrm{C}$. With such conditions, hydrogen concentrations into the optical fiber core reach more than $95 \%$ of the saturation level in the fiber core after $330 \mathrm{~h}$ (around 13 days).

Selected pressure condition accelerates hydrogen diffusion. We also performed measurement during natural hydrogen release at the end of the experiment: 16 days (versus 13 days under pressure) were required for exposed optical fibers to retrieve their original characteristics.

During hydrogen loading, samples were removed from autoclave regularly to perform measurements: distributed Brillouin scattering and absorption losses on a large wavelength span, from $1.1 \mu \mathrm{m}$ to $1.56 \mu \mathrm{m}$.

Measured spectral attenuations confirmed three absorption bands appear in the attenuation spectra of exposed optical fibers, the most important at $1245 \mathrm{~nm}$ and two smaller at $1165 \mathrm{~nm}$ and $1130 \mathrm{~nm}$, consistently with [17]. Losses reached $70 \mathrm{~dB} / \mathrm{km}$ (resp. $50 \mathrm{~dB} / \mathrm{km}$ ) at saturation at $1550 \mathrm{~nm}$ (resp. $1310 \mathrm{~nm}$ ). Standard Brillouin instruments have optical budget in the order of $10 \mathrm{~dB}$. As a result, in instrumented disposal cells for long-lived nuclear wastes, maximal distance range would significantly diminish with the increase of hydrogen content in the atmosphere, from the kilometer range down to one hundred meters. Improvement could be expected if working wavelength was tuned down to $1.3 \mu \mathrm{m}$.

Brillouin spectra before and after hydrogen exposure are illustrated in Figure 8 for the G652 fiber.

Brillouin scattering is modified by hydrogen content in optical fibers. On top of reduced amplitude, Brillouin scattering shifts towards high frequencies. This shift is somewhat linear and reaches $21 \mathrm{MHz}$ at saturation for the G652 and the F-doped fibers, $18 \mathrm{MHz}$ for the $\mathrm{HGe}$ fiber. Assuming standard coefficients $\left(C_{T}=1 \mathrm{MHz} /{ }^{\circ} \mathrm{C}, C_{\varepsilon}=0.05 \mathrm{MHz} / \mu \varepsilon\right)$, such a shift would induce an error in temperature (resp. strain) measurement in the order of $21^{\circ} \mathrm{C}$ (resp. $\left.420 \mu \mathrm{m} / \mathrm{m}\right)$.

Fluorine fiber is revealed to be more sensitive to small and moderate hydrogen contents than other fibers. 


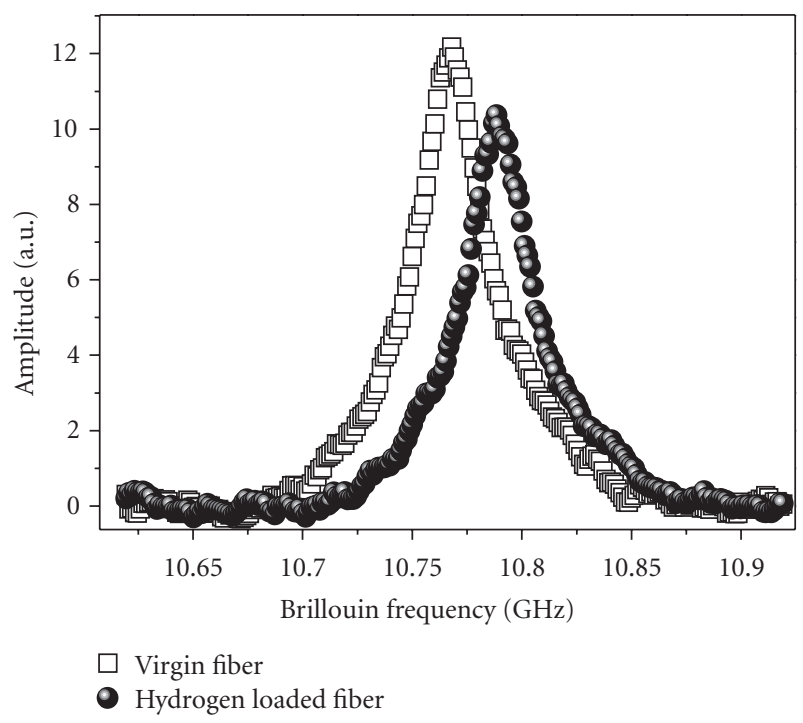

Figure 8: Measured Brillouin spectra in the G652 fiber before and after 13 days exposure to hydrogenated atmosphere (at saturation level).

At saturation, F-doped fiber and G652 are similar; HGe fiber is slightly less impacted. Further work is required to conclude if dopant type, internal stress, or defect concentrations are the major cause, or if differences are induced by different diffusion rates.

This result is not in favor of the application requirements since the F-doped fiber was the only sample which could endure radiations. In view of our application, we tested carbon coating which is known to prevent hydrogen migration into silica [18]. Samples endured the same hydrogen exposure. As detailed in [19], carbon coating of G652 fibers proved to be fully efficient since neither variation of propagation losses nor Brillouin frequency shift could be detected along these samples.

As a conclusion, an F-doped fiber with carbon coating is mandatory for the monitoring of the future geological repository where there will be both gamma radiation and hydrogen release.

4.3. Gamma Influence on Raman Sensing. Unlike Brillouin sensing which is wavelength encoded, for Raman sensing phenomena, RIA not only degrades distance range, but also induces measurement error. Indeed, temperature values are obtained by taking the ration of the two Raman components (Antistokes and Stokes) as detailed in (3) [20, 21]:

$$
\frac{I_{A S}}{I_{S}}=\left(\frac{\lambda_{S}}{\lambda_{A S}}\right)^{4} \exp \left(\frac{-h * \Delta v}{k * T(z)}-\int_{0}^{z}\left[\alpha_{A S}(\xi)-\alpha_{S}(\xi)\right] d \xi\right)
$$

where $\lambda_{A S}$ and $\lambda_{S}$ are Raman Antistokes and Stokes wavelengths, $\Delta v=13.2 \mathrm{THz}$ for silica, $h$ is the Planck's constant, $c$ is the speed of light in vacuum, $k$ is Boltzmann's constant, $T$ is the temperature of the optical fiber, and $\alpha$ refers to propagation losses.

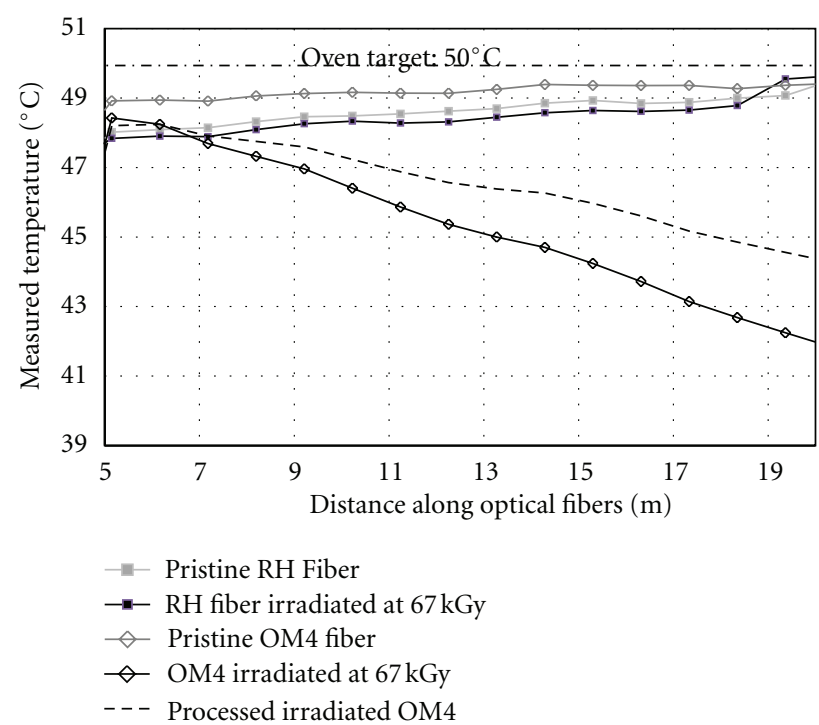

FIGURE 9: Temperature measurements based on Raman scattering in OM4 and radiation-hard fibers, pristine, and irradiated samples placed in an oven at $50^{\circ} \mathrm{C}$.

Gamma radiation induces differential losses at Stokes and Antistokes wavelengths, which then induce temperature measurement errors, consistently with (3), as detailed in $[20,21]$. Promoted solutions were to use double-ended configuration (closed loop).

However, single-ended arrangement (open loop) suits geological repository specification much better since (i) waste disposal cells are not always accessible at both ends and (ii) ability to perform measurements up to breaking points is important for durability in the order of a century. This is why we turned towards single-ended configuration and chose to focus on radiation-hard optical fibers.

Multimode silica-based fibers with $50 \mu \mathrm{m}$ core diameters were selected: standard (OM4 type) and radiation-hard fibers. Two samples were extracted from each optical fiber coil; one has been left pristine, the second sample has been irradiated.

We used a Co source at a dose rate of $0.65 \mathrm{kGy} / \mathrm{h}$. A $67 \mathrm{kGy}$ total dose was deposited in two steps. Postmortem temperature measurements were performed on pristine and irradiated samples of the two optical fiber types, placed inside a climatic chamber. Results are illustrated in Figure 9 for the $50^{\circ} \mathrm{C}$ temperature step.

Temperature measurements performed on standard pristine fiber matches imposed oven temperature $\left(50^{\circ} \mathrm{C}\right)$ with a $1^{\circ} \mathrm{C}$ shift. Once irradiated, large measurement errors appear; error increases with distance, up to $10^{\circ} \mathrm{C}$ error cumulated in $20 \mathrm{~m}$. OTDR (optical time-domain reflectometer) measurements revealed $80 \mathrm{~dB} / \mathrm{km}$ at $850 \mathrm{~nm}$ and $21 \mathrm{~dB} / \mathrm{km}$ at $1300 \mathrm{~nm}$. As shown in Figure 9, raw data may be processed to take into account this $0.06 \mathrm{~dB} / \mathrm{m}$ differential loss value, applying (3). It limits significantly temperature error, but linear drift still remains. Since Raman instrument works at $1064 \mathrm{~nm}$, propagation losses measurements should rather be performed at the real Stokes and Antistokes wavelengths, $1110 \mathrm{~nm}$ and $1020 \mathrm{~nm}$. It will be performed in a near future. 


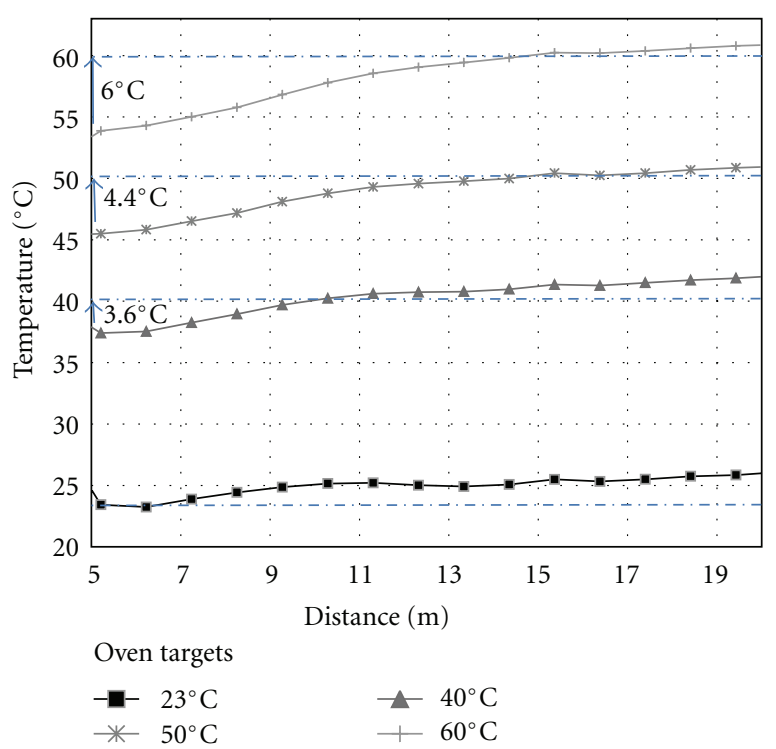

Figure 10: Temperature measurements based on Raman scattering in the radiation-hard fiber irradiated at $618 \mathrm{kGy}$ total dose.

Pristine as well as irradiated radiation hard-fiber samples provide successful temperature measurements (Figure 9), however, with poor accuracy, in the order of $3^{\circ} \mathrm{C}$.

We increased irradiation dose. Second irradiation run was performed in MOL facilities, simultaneously with singlemode fibers. It consisted in gamma rays, with a dose of $1,5 \mathrm{kGy} / \mathrm{h}$ during 341 hours. Total dose of 551,5 kGy was thus deposited on top of the previous $67 \mathrm{kGy}$, which provided $618 \mathrm{kGy}$. Sample was submitted to temperature changes, $23^{\circ} \mathrm{C}, 40^{\circ} \mathrm{C}, 50^{\circ} \mathrm{C}$, and $60^{\circ} \mathrm{C}$. As illustrated in Figure 10, temperature sensing remains possible even after $0.6 * 10^{7} \mathrm{~Gy}$ value that approaches a hundred years of monitoring in the geological repository.

Similarly, with the pristine radiation-hard fiber, temperature measurement quality is poor, degraded by (i) a bias to the oven temperature value and (ii) a linear drift that increases with distance. This linear error is positive, whereas it was negative for standard fiber. Indeed, measured losses at $850 \mathrm{~nm}$ and $1300 \mathrm{~nm}$ were, respectively, $51 \mathrm{~dB} / \mathrm{km}$ and $74 \mathrm{~dB} / \mathrm{km}$ (larger at $1300 \mathrm{~nm}$ ); thus, differential losses are opposite to previous case.

4.4. Hydrogen Influence on Raman Scattering. Hydrogen influence on Raman distributed temperature sensing has been described in the literature [22] and is expected to induce differential losses, thus temperature reading errors similar with radiation effects. Based on hydrogen tests performed with single-mode fibers (Section 4.2), Andra will supply carbon-coated multimode radiation-hard fibers in the future.

4.5. Discussion. In order to predict distributed temperature and strain measurement performances in the future geological repository for long-lived nuclear waste, we considered separately gamma radiation and hydrogen influences on Brillouin and Raman scatterings in various optical fiber types.
However, combined influence of hydrogen and radiation is expected to be in favor of measuring performances [23]. Presented results are worst cases.

Carbon coating should also be in favor of radiation tolerance.

Temperature, in the order of $90^{\circ} \mathrm{C}$ in the vicinity of highlevel waste disposal cell, will accelerate hydrogen diffusion in optical fibers [24]; for ease of manipulation, pressure, instead of temperature, was used in the test. Equivalence will need to be checked.

Tests on radiation influence on Raman sensing were limited to postmortem measurements. These preliminary results will be augmented with on-line measurements on hydrogenloaded carbon-coated radiation-hard fiber. Spectral transmission measurements will also be included to take full advantage of the compensation model.

\section{Conclusion}

We developed a strategy to design a durable monitoring system for nuclear structures, based on truly-distributed optical fiber sensors.

We demonstrated Brillouin strain measurement can be efficiently compensated from temperature influence installing a parallel sensing line paired with a Raman instrument.

State-of-the-art results on gamma and hydrogen influences on Rayleigh, Raman, and Brillouin scatterings were obtained. Fluorine-doped pure-silica-core fibers coated with carbon will be mandatory to handle high gamma dose $\left(10^{7} \mathrm{~Gy}\right)$ and hydrogen release in the vicinity of high-level long-lived waste disposal cells. After a century of monitoring based on Brillouin scattering, maximal range would decrease down to a hundred meter but strain measurement uncertainty should remain stable. With other fiber types or primary coatings, large strain measurement errors would ruin the monitoring system reliability.

To reduce hydrogen and radiation influences, a significant future improvement could be achieved by choosing the operating wavelength at $1.3 \mu \mathrm{m}$ instead of $1.55 \mu \mathrm{m}$.

We showed that radiation-hard fibers enabled single-end Raman measurements up to 0.6 MGy; however, temperature measurement uncertainty, presently in the order of few degrees, must be improved. A postprocessing to compensate for differential loss induced by radiation has been proposed and will, in the future, take benefit of spectral measurements.

Next step of Andra qualification procedure is to incorporate these special fibers into strain sensing cables and run (i) combined hydrogen-gamma influence test and (ii) another outdoor test, to evaluate if curvatures or splicing may get more sensitive than with standard fibers.

\section{Acknowledgments}

Part of this work has been done in the frame of the COST Action TD1001: Novel and Reliable Optical Fibre Sensor Systems for Future Security and Safety Applications (OFSeSa). D. Sporea acknowledges that this work was partially supported by the grant of the Romanian National 
Authority for Scientific Research, CNDI-UEFISCDI, project 8/2012 "Sensor Systems for Secure Operation of Critical Installations."

\section{References}

[1] J. M. Lopez-Higuera, Handbook of Optical Fiber Sensing Technology, Wiley, New York, NY, USA, 2002.

[2] A. Rogers, "Distributed optical-fibre sensing," Measurement Science and Technology, vol. 10, no. 8, pp. R75-R99, 1999.

[3] V. Lanticq, R. Gabet, F. Taillade, and S. Delepine-Lesoille, "Distributed optical fibre sensors for structural health monitoring: upcoming challenges," in Optical Fibre, New Developments, L. Christophe, Ed., chapter 9, pp. 177-199, InTech, Olajnica, 2010.

[4] "Qualification guide FD CEN/TR, 14748," Non-destructive testing-methodology for qualification of non-destructive tests, 2005.

[5] S. W. Tyler, J. S. Selker, M. B. Hausner et al., "Environmental temperature sensing using Raman spectra DTS fiber-optic methods," Water Resources Research, vol. 45, Article ID W00D23, 11 pages, 2009.

[6] S. Delepine-Lesoille, J. M. Henault, G. Moreau et al., "Truly distributed optical fiber sensors for structural health monitoring: From the telecommunication optical fiber drawling tower to water leakage detection in dikes and concrete structure strain monitoring," Advances in Civil Engineering, vol. 2010, Article ID 930796, 13 pages, 2010.

[7] M. Niklès, L. Thévenaz, and P. A. Robert, "Simple distributed fiber sensor based on Brillouin gain spectrum analysis," Optics Letters, vol. 21, no. 10, pp. 758-760, 1996.

[8] M. Niklès, L. Thévenaz, and P. A. Robert, "Brillouin gain spectrum characterization in single-mode optical fibers," Journal of Lightwave Technology, vol. 15, no. 10, pp. 1842-1851, 1997.

[9] J. P. Dubois, S. Delepine-Lesoille, V. H. Tran et al., "Raman versus Brillouin optical fiber distributed temperature sensing: an outdoor comparison (metallic beam and concrete slab)," in Proceedings of the 4th International Society for Structural Health Monitoring of Intelligent Infrastructures Conference (SHMII '09), July 2009.

[10] V. Lamour, A. Haouas, J. P. Dubois, and R. Poisson, "Long term monitoring of large massive concrete structures: cumulative effects of thermal gradients," in Proceedings of 7 th International Symposium on Nondestructive Testing in Civil Engineering, Nantes, France, July 2009.

[11] M. N. Alahbabi, Y. T. Cho, and T. P. Newson, "Simultaneous temperature and strain measurement with combined spontaneous Raman and Brillouin scattering," Optics Letters, vol. 30, no. 11, pp. 1276-1278, 2005.

[12] D. Sporea, A. Sporea, S. O'Keeffe, D. McCarthy, and E. Lewis, "Optical fibers and optical fiber sensors used in radiation monitoring," in Selected Topics on Optical Fiber Technology, Y. Moh, S. W. Harun, and H. Arof, Eds., Intech, Vienna, Austria, 2012.

[13] M. Van Uffelen, Modélisation de systèmes d'acquisition et de transmission à fibres optiques destinés à fonctionner en environnement nucléaire [Ph.D. thesis], Université de Paris, Orsay, France, 2001.

[14] D. Alasia, A. Fernandez Fernandez, L. Abrardi, B. Brichard, and L. Thévenaz, "The effects of gamma-radiation on the properties of Brillouin scattering in standard Ge-doped optical fibres," Measurement Science and Technology, vol. 17, no. 5, pp. 1091-1094, 2006.

[15] X. Pheron, Y. Ouerdane, S. Girard et al., "In situ radiation influence on strain measurement performance of Brillouin sensors," in Proceedings of the 21st International Conference on Optical Fiber Sensors, May 2011.

[16] A. Fernandez-Fernandez, H. Ooms, B. Brichard et al., "SCKCEN gamma irradiation facilities for radiation tolerance assessment," in Proceedings of the NSREC Data Workshop, pp. 171-176, 2002, 02HT8631.

[17] J. Stone, "Interactions of hydrogen and deuterium with silica optical fibers," Journal of Lightwave Technology, vol. 5, no. 5, pp. 712-733, 1987.

[18] P. J. Lemaire and E. A. Lindholm, "Hermetic optical fibers: carbon coated fibers," in Specialty Optical Fibers Handbook, A. Mendez and T. F. Morse, Eds., pp. 453-490, New York Academic, 2007.

[19] S. Delepine-Lesoille, J. Bertrand, L. Lablonde, and X. Phéron, "Distributed hydrogen sensing with Brillouin scattering in optical fibers," Photonics Technology Letters, vol. 24, no. 17, pp. 1475-1477, 2012.

[20] F. B. H. Jensen, E. Takada, M. Nakazawa, T. Kakuta, and S. Yamamoto, "Consequences of radiation effects on pure-silicacore optical fibers used for raman-scattering-based temperature measurements," IEEE Transactions on Nuclear Science, vol. 45, no. 1, pp. 50-58, 1998.

[21] A. Fernandez Fernandez, P. Rodeghiero, B. Brichard et al., "Radiation-tolerant Raman Distributed Temperature monitoring system for large nuclear infrastructures," IEEE Transactions on Nuclear Science, vol. 52, no. 6, pp. 2689-2694, 2005.

[22] C. Martelli, A. L. C. Triques, A. Braga et al., "Operation of optical fiber sensors in hydrogen-rich atmosphere," in Proceedings of the 4th European Workshop on Optical Fibre Sensors, September 2010.

[23] B. Brichard, A. L. Tomashuk, V. A. Bogatyrjov et al., "Reduction of the radiation-induced absorption in hydrogenated pure silica core fibres irradiated in situ with $\gamma$-rays," Journal of Non-Crystalline Solids, vol. 353, no. 5-7, pp. 466-472, 2007.

[24] P. J. Lemaire, "Reliability of optical fibers exposed to hydrogen: prediction of long-term loss increases," Optical Engineering, vol. 30, no. 6, pp. 780-789, 1991. 

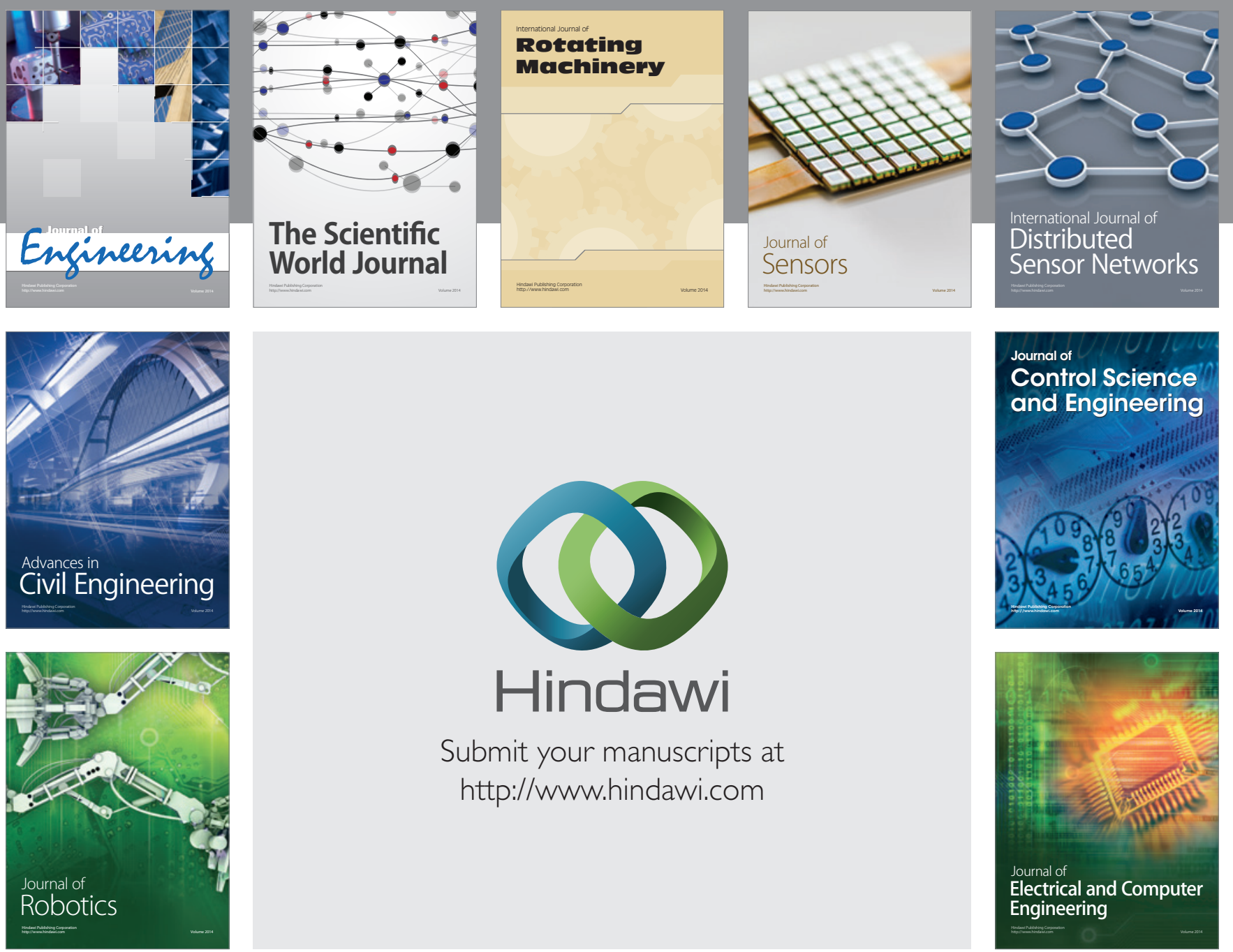

Submit your manuscripts at

http://www.hindawi.com
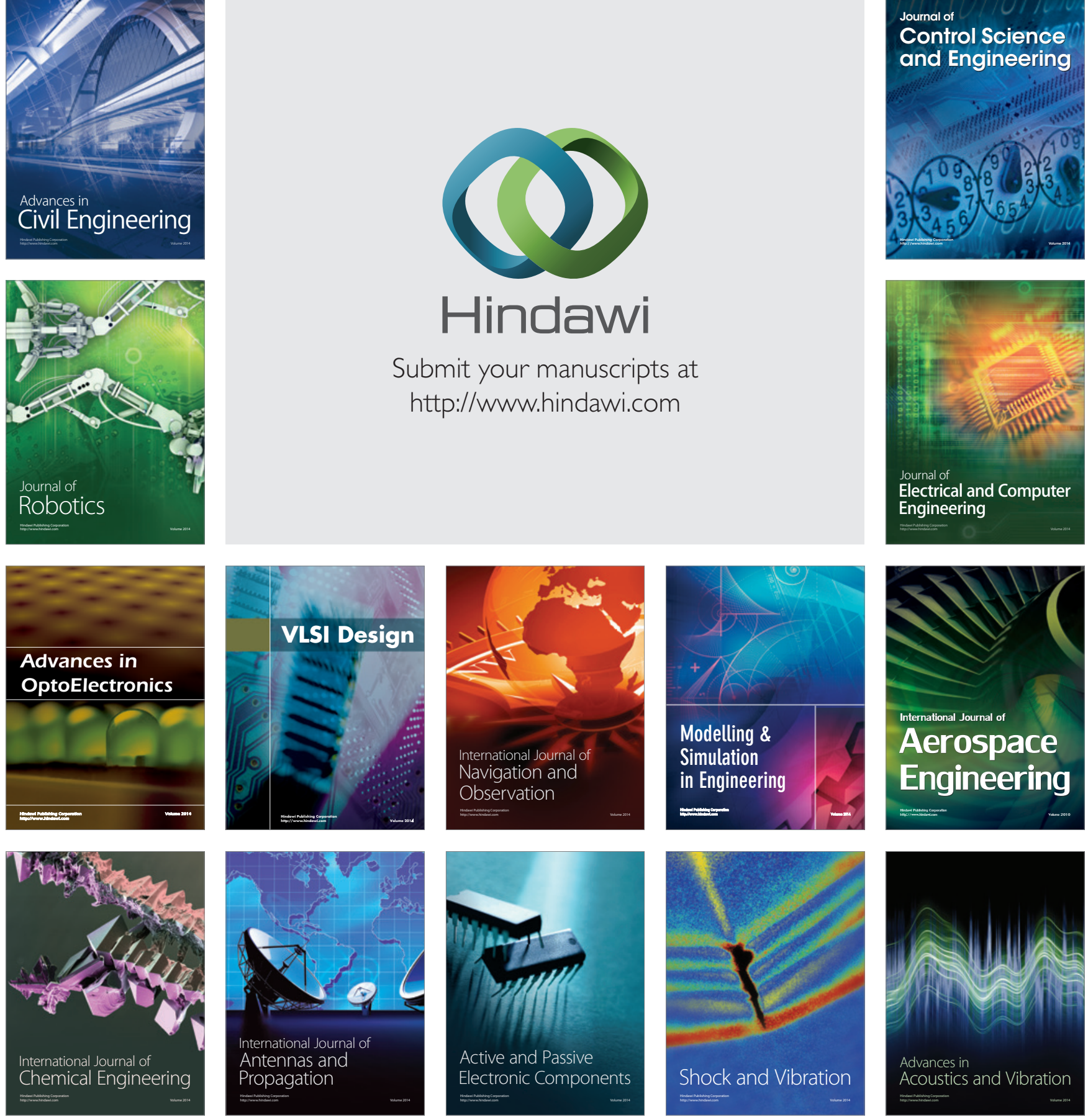